\title{
Influence of the absorption coefficient in Nd laser annealing of amorphized semiconductor layers
}

\author{
M. von Allmen a) and W. Lüthy

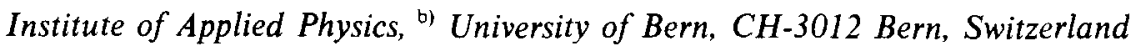 \\ J. P. Thomas, M. Fallavier, J. M. Mackowski, R. Kirsch, and M-A. Nicolet'
}

Institut de Physique Nucléaire et $I N_{2} P_{3}, F-69621$ Villeurbanne, France

M. E. Roulet

Centre Electronique Horloger, ${ }^{\text {b) }} \mathrm{CH}-2000$, Neuchatel, Switzerland

(Received 25 August 1978; accepted for publication 12 October 1978)

It is shown that epitaxial regrowth of thin amorphized Si layers by Nd-laser irradiation is strongly affected by temperature-induced changes in the absorptivity of the crystalline substrates. This results in an amplification of small spatial variations of the absorbed intensity by local thermal runaway. The problem can be avoided by either preheating the sample or by applying pulses of long duration and reduced intensity. A model explaining the observations is proposed.

PACS numbers: 79.20.Ds, $68.55 .+$ b, 81.40.Ef, $42.60 . \mathrm{Kg}$

The use of pulsed Nd: YAG lasers to induce epitaxial regrowth of amorphized $\mathrm{Si}$ layers has been studied by several workers. $^{i-5}$ The irradiation with Nd-laser pulses reveals an unusual sensitivity of silicon to small lateral inhomogeneities of the intensity distribution or the irradiated material..$^{3.4}$ In this letter we report on experiments to clarify this behavior and its influence on epitaxial regrowth.

Amorphous Si layers approximately $2100 \AA$ A thick were produced by implantation of ${ }^{28} \mathrm{Si}^{+}$at a dose of $10^{15}$ ions $/ \mathrm{cm}^{2}$ and an energy of $100 \mathrm{keV}$ into $150-\Omega \mathrm{cm} n$-type $\langle 100\rangle \mathrm{Si}$ wafers at $\mathrm{LN}_{2}$ temperature. The layers were shown to regrow epitaxially under thermal annealing at $550^{\circ} \mathrm{C}$ for $2 \mathrm{~h}$. The amorphized samples were irradiated in air with 30-ns pulses from a $Q$-switched Nd : YAG laser. The radial intensity distribution of the laser beam was nearly Gaussian with a small annular modulation due to diffraction at a circular aperture. Figure 1 show a cross section of the beam spot displayed with the aid of a linear photodiode array. $2-\mathrm{MeV}^{4} \mathrm{He}$ backscattering spectrometry (BS) and channeling along the $\langle 100\rangle$ direction were performed to establish the amorphous state of the implanted layer and the epitaxial regrowth induced by the laser beam.

Figure 2 shows a Nomarski interference micrograph of a sample irradiated at room temperature with a $Q$-switched pulse of $33 \mathrm{MW} / \mathrm{cm}^{2}$. Figure 2 shows a large enhancement of the beam inhomogeneities. The associated BS spectrum does not detectably differ from that of the as-implanted layer. Increasing the power resulted in further enhancement of the lateral nonuniformities in the irradiated area.

Present address: California Institute of Technology, Department of Electrical Engineering, Pasadena, Calif. 91125.

"'Work supported in part by the Swiss Commission for the Encouragement of Scientific Research.

"On leave from California Institute of Technology, Pasadena. Calif, 91125.
The demonstrated amplification of beam-spot inhomogeneities is related to the fact that the Nd-laser photon energy $(1.17 \mathrm{eV})$ is close to the indirect Si energy gap. At room temperature the pertaining absorption coefficient is on the order of $10 \mathrm{~cm}^{-1}$ in crystalline Si and strongly increases with temperature. ${ }^{6}$ Although the absorption coefficient of the amorphous layer is on the order of $10^{4} \mathrm{~cm}^{-1}$, ? a layer of 2000 $\AA$ absorbs only about $20 \%$ of the incident radiation. Heating is therefore dominated by light absorbed in the substrate. Here, the increase of the absorption coefficient provides a positive feedback that tends to make the heating process inherently unstable. If an inhomogeneity of either the laser spot or the wafer material causes the instantaneous heat imput to rise slightly at a given point in the irradiated sample, this induces a local temperature rise. The increase of the local absorption coefficient in turn causes the heat input to increase even more.

We found two different experimental possibilities to overcome the described difficulty. The first consists of keep-

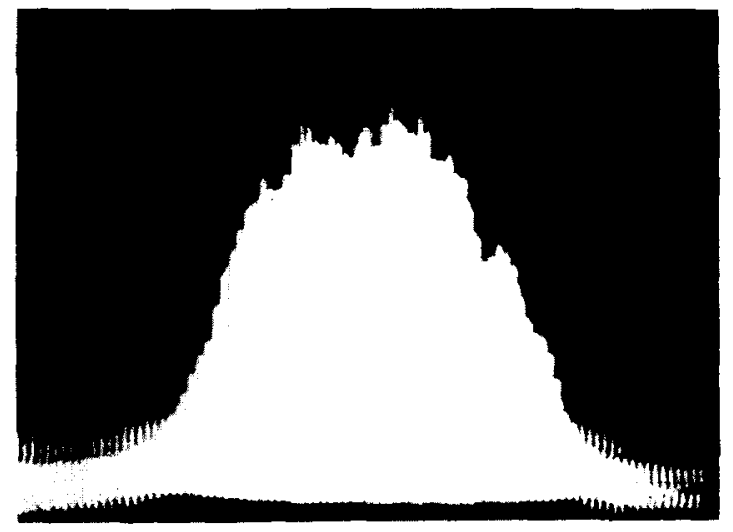

FIG. 1. Cross section of the intensity profile of the $Q$-switched Nd laser pulse monitored by a linear photodiode array. 


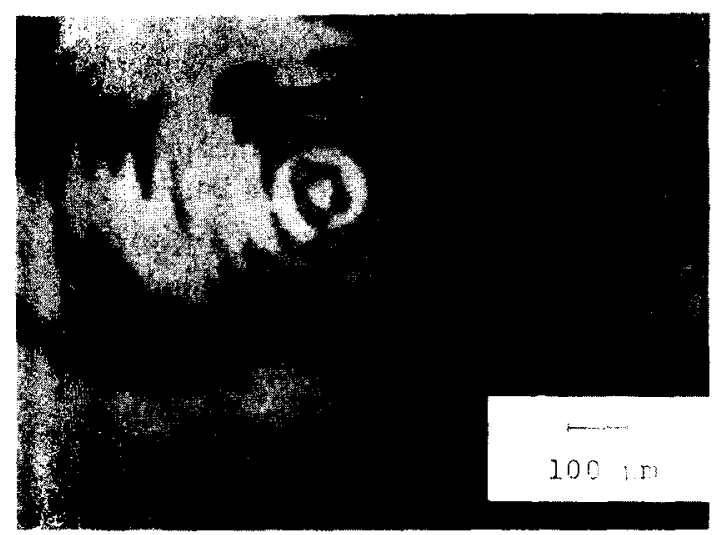

FIG. 2. Nomarski interference micrograph of a sample surface irradiated at $20{ }^{\circ} \mathrm{C}$ with a $Q$-switched pulse of $1.0 \mathrm{~J} / \mathrm{cm}^{2}$ (central portion of the laser impact area)

ing the samples at an elevated temperature during laser irradiation. At a high sample temperature the initial absorption is increased uniformly. Figure 3(a) shows a micrograph of a sample irradiated by the same laser pulse as in Fig. 2, but at an initial temperature of $250^{\circ} \mathrm{C}$. In this case, the irradiated area is laterally uniform. The BS spectra [Fig. 3(b)] indicate fairly good epitaxial regrowth.

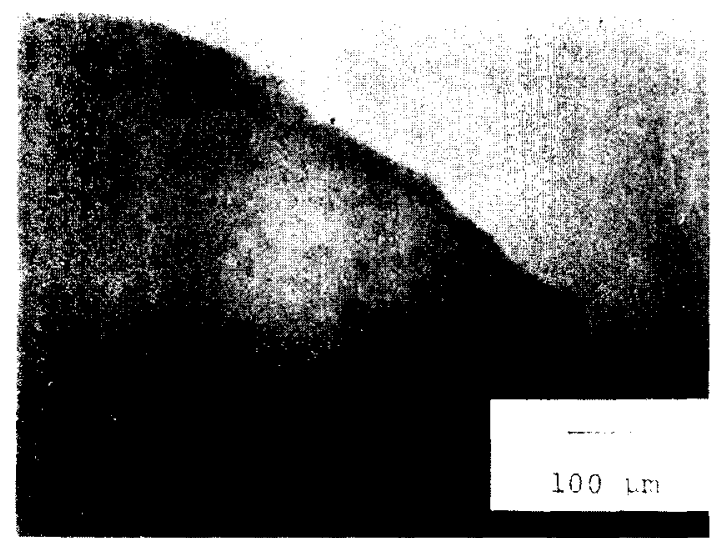

(a)

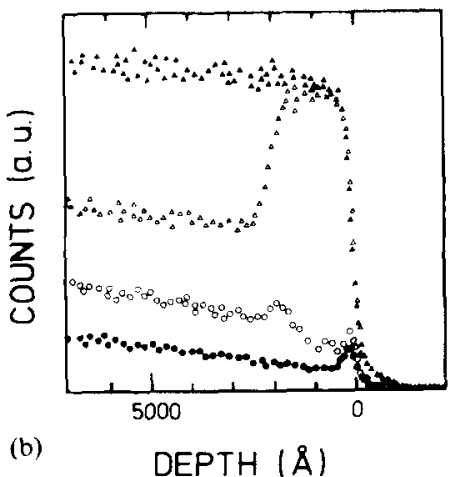

FIG. 3. (a) Micrograph of a sample surface irradiated at $250^{\circ} \mathrm{C}$ with the same pulse as in Fig. 2 (peripheral portion of the laser impact area). (b) Spectra of 2.0. $\mathrm{MeV}^{4} \mathrm{He}^{+}$particles backscattered from the central portion of the laser impact area: $\Delta$ random and $\langle 100\rangle$-aligned incidence on unimplanted sample; $\langle 100\rangle$-aligned incidence on implanted sample $\Delta$ before and $O$ after laser irradiation.

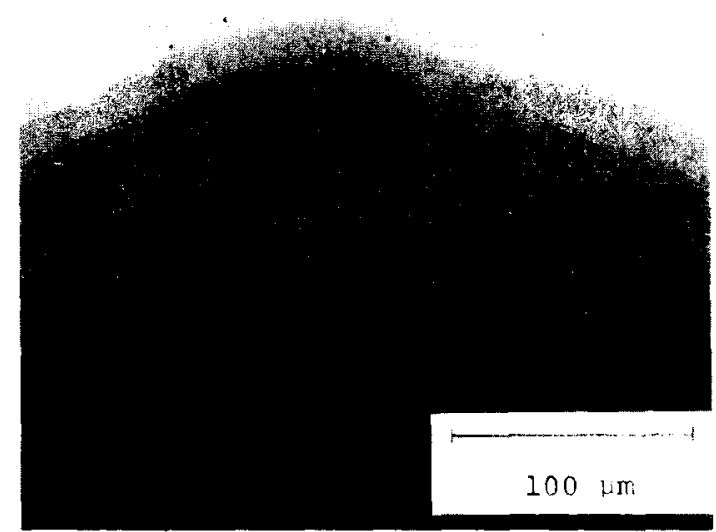

(a)

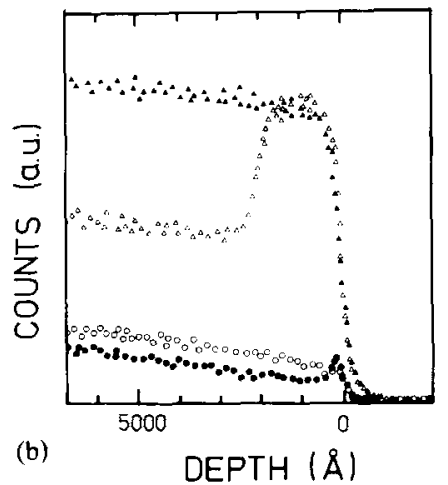

FIG. 4. Data similar to those shown in Fig. 3. The micrograph shows the periphery of an individual laser impact area. The irradiation is with partially overlapping $100-\mu$ s pulses of $300 \mathrm{~kW} / \mathrm{cm}^{2}$ gated from a free-running $\mathrm{Nd}$ laser.

Another possible way to avoid nonhomogenous heating consists of applying pulses of increased duration and reduced power. We used pulses from a free-running Nd : YAG laser with an external pulse-shaping facility. The temporal pulse shape was rectangular and the pulse duration was 100 $\mu \mathrm{s}$. The spot size was about $1 \mathrm{~mm}$. Many overlapping irradiations were used to scan an extended area. There was no indication of local thermal runaway. Figure 4(a) shows a micrograph of part of the irradiated zone. The characteristic surface pattern has been described previously. ${ }^{4}$ The associated BS spectra [Fig. 4(b)] show epitaxial regrowth of the scanned area.

Our interpretation of the experimental observations is based on the following ideas: (i) The temperature distribution produced in a solid by absorption of light depends on the relative magnitude of the absorptionlength $\alpha^{-1}$ and the diffusivity length $(D t){ }^{1 / 2}$ Here, $t$ is the time and $D$ is the thermal diffusivity. If the absorption length is large $\left[\alpha^{-1} \gg(D t)^{1 / 2}\right.$, regime I], diffusion is negligible and the temperature at any time and point is determined by the absorbed energy. If, however, the absorption length is short $\left[\alpha^{-1} \ll(D t)^{1 / 2}\right.$, regime II], diffusion governs the temperature distribution. (ii) The absorption length $\alpha^{-1}$ strongly decreases with temperature. (iii) The Si surface reaches the melting point. When this occurs, the temperature stops rising due to a substantial increase in reflectivity ${ }^{4}$ and to absorption of latent heat. These conditions lead to the following sequence of events. 
With a sample at room temperature, the initial absorption length is large. Correspondingly, most of the pulse energy is spent on slowly heating the sample over an appreciable depth (regime I). The consequent decrease in absorption length accelerates the rate at which the temperature rises. Eventually, the temperature rises very abruptly and regime II is reached. During regime I the energy deposited at each point is cumulative, i.e., a spot of enhanced intensity in the laser beam causes the same sequence of events to develop proportionally faster and surface melting to occur sooner. Lateral nonuniformities thus arise before diffusion becomes significant.

With a sample at elevated temperature, the initial depth heated in regime $I$ is much shorter. The energy required to reach regime II is smaller, and most of the pulse energy is spent on heating the surface region, regardless of lateral intensity variations. Surface melting sets in early everywhere. Lateral nonuniformities are attenuated at this point by the increased reflectivity of the melt, by the halt in the temperature rise associated with the latent heat absorption, and by diffusion. The essential difference as compared to the unheated sample is that most of the pulse energy is spent in regime II, where intensity variations cause only minor local differences in the evolution of temperature with time.

Regarding the experiment with $100-\mu$ s pulses, the absence of thermal runaway can be explained by diffusion which smoothes out local variations in absorption length. In terms of the previous discussion, this means that regime II is dominant.
To conclude, we show that Nd-laser annealing of thin amorphous $\mathrm{Si}$ layers is strongly affected by temperature-induced changes of absorptivity. The resulting thermalrunaway problems can be overcome by either preheating the sample or by using long pulses of reduced intensity.

We thank E. Rimini (Catania), S.S. Lau, S. Matteson, and $\mathrm{G}$. Chapman (Pasadena) for providing samples. We also thank the atomic collision group of the IPN in Lyon for assistance in the channeling experiments and H.P. Weber (Bern) for his stimulating interest.
'I.B. Khaibullin, E.I. Shtyrkov, M.M. Zaripov, R.M. Bayazitov, and M.F.
Galyatudinov, Radiat. Eff. 36, 225 (1978).
${ }^{2}$ G.K. Celler, J.M. Poate, and L.C. Kimerling, Appl. Phys. Lett. 32, 464
(1978).
'H.J. Leamy, G.A. Rozgonyi, T.T. Sheng, and G.K. Celler, Appl. Phys.
Lett. 32, 535 (1978).
'M. von Allmen, W. Lüthy, and K. Affolter, Appl. Phys. Lett. 33, 824
(1978).
J.C. Müller, A. Grob, J.J. Grob, R. Stuck, and P. Siffert (unpublished).
${ }^{6}$ For illustration, we measured the absorption coefficient $\alpha$ at $\lambda=1.06 \mu \mathrm{m}$ of
monocrystalline $n$-type Si $(4 \Omega \mathrm{cm})$ and found an increase from 36 to 335
$\mathrm{~cm}^{-1}$ in the temperature range $20-330^{\circ} \mathrm{C}$. The values follow an exponential
law.
'A measurement of an amorphous film produced by evaporation of $6000 \AA$
of Si onto a glass substrate gave $\alpha(1.06 \mu)=0.9 \times 10^{4} \mathrm{~cm}^{-1}$ at room tempera-
ture. Up to $350^{\circ} \mathrm{C}$, the temperature dependence was insignificant.

\title{
A computer simulation of laser annealing silicon at $1.06 \mu \mathrm{m}$
}

\author{
J. C. Schultz ${ }^{\text {a) }}$ and R. J. Collins
}

Department of Electrical Engineering, University of Minnesota, Minneapolis, Minnesota 55455 (Received 15 September 1978; accepted for publication 24 October 1978)

\begin{abstract}
A computer simulation of laser annealing ion-implanted silicon with $1.06-\mu \mathrm{m}$ radiation at energy densities of $38-76 \mathrm{MW} / \mathrm{cm}^{2}$ shows that the amorphous surface layer of the silicon melts. Quantitative predictions for the depth of the molten zone and the impurity distribution as a function of peak laser intensity and pulse duration agree well with published experimental observations. Additional analysis indicated that pulse duration, peak power density, and the impurity implantation depth are of primary importance in controlling the laser annealing process. Also, step junctions may be produced for optimal values of these parameters.
\end{abstract}

PACS numbers: $61.80 . J h, 79.20 . \mathrm{Ds}, 81.10 .-\mathrm{h}, 81.40 . \mathrm{Ef}$

The use of high-intensity laser pulses to anneal surface damage caused by the ion-implantation process has been shown to have significant advantages over the traditional thermal annealing methods. ${ }^{1-3}$ However, models of trans- port processes within solid silicon are unable to produce any mechanism capable of causing such changes, ${ }^{2}$ and, consequently, a liquid region on the irradiated surface has been postulated. ${ }^{i}$ To verify the existence of such a molten zone, a computer simulation was carried out for heat and impurity diffusion in silicon irradiated by $1.06-\mu \mathrm{m}$ radiation. The computer simulation included the temperature dependence

"Supported in part by funds from the National Science Foundation. 